
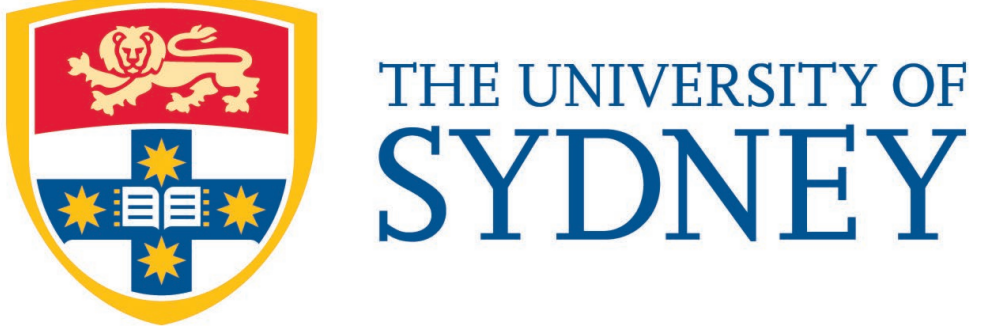

Economics Working Paper Series

$2018-5$

Blocking in a timing game with asymmetric players

Vladimir Smirnov and Andrew Wait

May 2019 


\title{
Blocking in a timing game with asymmetric players*
}

\author{
Vladimir Smirnov and Andrew Wait ${ }^{\dagger}$
}

May 14, 2019

\begin{abstract}
We examine innovation as a market-entry timing game with complete information and observable actions. We allow for heterogenous payoffs between players, and for a leader's payoff functions to be multi-peaked and nonmonotonic. Assuming that the follower's payoff is non-increasing with the time of the leader's entry, we characterize all pure-strategy subgame perfect equilibria for the two-player asymmetric model, showing that there are at most two equilibria. Firm heterogeneity allows for equilibria with different characteristics than previously examined in the literature. For example, a firm may wish to enter earlier blocking its rival's entry, so as to avoid an anticipated lower future payoff if it waited. A notable feature of this blocking equilibrium is that rents need not be equalized between the leader and follower. We also show that if the followers' payoffs are non-monotonic, the iterative incentives to block each other's product launch may lead to starkly inefficient early entry in a continuous version of the centipede game.

Key words: timing games, blocking entry, innovation.

JEL classifications: C72, L13, O31, O33.
\end{abstract}

*We would like to thank Murali Agastya, Simon Board, Nicolas de Roos, Simon Grant, Sander Heinsalu, Suraj Prasad, Kunal Sengupta and participants at the Organizational Economics Workshop 2017, University of Sydney and Microeconomic Theory Workshop 2017, Victoria University of Wellington and seminars at Kyoto University and the Higher School of Economics. The authors are responsible for any errors.

† email: vladimir.smirnov@sydney.edu.au; andrew.wait@sydney.edu.au. School of Economics, University of Sydney, NSW 2006 Australia. 


\section{Introduction}

When should a firm innovate or launch a new product? Sometimes it is better to be first into a market. Reinganum (1981a,b) shows that when firms can't observe their rival's actions (in an open-loop equilibrium), the leader can receive a higher payoff than the follower, who may enter much later even though the duopolists are ex ante identical. A leader, of course, need not always be better off; Fudenberg and Tirole (1985) show that when rivals' actions are observable (in a closed-loop equilibrium) the incentive to preempt can dissipate all potential gains from entering first, equalizing rents to both firms in the process. Moreover, it might be advantageous to enter the market second, rather than first, as the market pioneer might need to incur set-up or R\&D costs on which its rival can free-ride.

Several themes run through this existing literature. Firstly, given the potential strategic interaction it brings, the presence of a rival(s) complicates a firm's entry decision. Secondly, this interaction can induce inefficient entry (Fudenberg and Tirole, 1985). Drawing on these themes we study a novel duopoly model of innovation that allows for: (i) heterogeneous firms; and (ii) the possibility that different technologies become available (at a later date) if a firm delays entry. We provide a general solution method and completely characterize the pure-strategy subgame perfect equilibria.

The basic features of our model are as follows. Two firms can make an irreversible and one-off decision to enter a market. Time is continuous and all previous actions (entry or not) are observable; consistent with this, we focus on closed-loop equilibria. In Fudenberg and Tirole (1985) and others, the entrants are ex ante identical and have access to the same potential innovation. But usually firms are not all the same. Drawing inspiration from Katz and Shapiro (1987), we study two heterogenous firms that can have different payoffs from entering at a given time. ${ }^{1}$ This assumption of heterogeneity is applicable in many situations. Firms

\footnotetext{
${ }^{1}$ Katz and Shapiro (1987) analyze an innovation game with heterogenous firms when there is licensing (by the leader) and imitation (by the follower). They find that industry leaders (who are more efficient) need not be the firm that innovates, as it may prefer to free ride on the public good provided by its rival. Riordan (1992) uses a similar framework to examine the impact of regulation of technological adoption. Also see Galasso and Tombak (2014), who adapt Katz and Shapiro (1987) to study the take-up of green technologies that have both a private and public
} 
might differ in their ability to exploit market opportunities. For example, expected payoffs from launching a new phone handset could differ between two rivals, given their preexisting reputation, network or tie-in products. The same can be said for a process (cost-saving) innovation - its payoff depends on access to markets, how the new technology meshes with a firm's existing practices, and so forth. As noted above, often firms also have to choose which technology to implement. Returning to the smartphone example, Samsung made a choice to switch its cell phone operating system from its own in-house system to an Android platform; Sony also made an equivalent choice. Despite its closed system, in many ways Apple faces a similar tradeoff when contemplating the timing of a new iOS for its devices.

Implicit in this is that not all technologies are available immediately; rather, some technologies are only available (or worth considering) later. This potentially changes the payoff structure - for example, unlike in Katz and Shapiro (1987), a leader's entry payoff can be multi-peaked with respect to entry time, reflecting when the new technology becomes available. This payoff structure, generated by the choice between multiple technologies, combined with the asymmetric payoffs between players, creates a new strategic entry environment not previously analyzed.

While we place effectively no restrictions on the leader's payoff function other than continuity, in a similar way to Hoppe and Lehmann-Grube (2005) and Argenziano and Schmidt-Dengler (2012, 2013, 2014) we assume that the payoff of the follower is non-increasing with leader's time of entry. This could be the case, for instance, when later entry by the leader affords it to enter the market with a better (less costly) production technology or product, or possibly both, which in turn exerts greater competitive pressure on the second entrant.

Adapting the solution technique of Smirnov and Wait (2015) to asymmetric firms, we characterize all pure-strategy subgame perfect equilibria for this game. In any entry game in our setting we find that there can be zero, one or two purestrategy equilibria. Sometimes the equilibria take a familiar form. Just like in Fudenberg and Tirole (1985) and Katz and Shapiro (1987), we show that there can be a preemption equilibrium, in which a firm enters before the stand-alone

good benefit by asymmetric firms. 
entry time. ${ }^{2}$ A second-mover advantage equilibrium is also possible; while both parties would like to be second, in equilibrium one of the two needs to self-sacrifice and enter first. ${ }^{3}$ Asymmetric payoffs, however, allow for other, more peculiar, possibilities. For example, a firm may wish to enter earlier (block it rival) so as to avoid a lower payoff that it would receive if it waited and allowed its rival to enter as the leader in the future. Anticipating this, the other firm might enter even earlier, effectively stymiing its rival's future blocking move. This discrete leapfrogging characteristic of firms opting to enter earlier so as to gazump their rival in what we call a blocking equilibrium is only possible with heterogenous firms. Moreover, rents need not be equalized in this blocking equilibrium. This type of equilibrium is relevant, capturing some of the interplay between technology companies such as the decision as to when to launch a new smartphone by rivals like Apple and Samsung (and others). ${ }^{4}$ In other situations still, asymmetric payoffs also allow for the possibility that no equilibrium in pure strategies exists at all.

As noted, the base model assumes that follower payoffs are non-increasing in the time of entry of the leader. Whilst it aids in exposition, this assumption is not necessary for our analysis. Section 3.2.3 explores the implications of relaxing this assumption. In particular, we consider the situation when the follower payoff curve is multi-peaked in a continuous market-entry version of the centipede game. This generates an iterative process in which each firm has an incentive to enter earlier, fearful of a lower payoff it could receive if its rival was allowed to enter as a leader later. In equilibrium, entry occurs inefficiently early. This outcome is indicative of the products being launched when they are really not ready, squandering opportunities for more mature and socially efficient innovation. ${ }^{5}$

\footnotetext{
${ }^{2}$ This terminology follows Katz and Shapiro (1987), in which the stand-alone entry time is time of entry a firm would choose if it faces no threat of entry by a rival.

${ }^{3}$ Theoretically, second-mover advantages with observable actions have been studied by Dutta et al. (1995), Hoppe (2000), Hoppe and Lehmann-Grube (2001), Hoppe and Lehmann-Grube (2005) and Smirnov and Wait (2007, 2015). Also see the empirical findings of Tellis and Golder (1996), who show that early imitators often outperform market pioneers.

${ }^{4}$ See 'Phone tag; Apple v Samsung' in The Economist, September 162017.

${ }^{5}$ This type of equilibrium is reminiscent of deterrence/accommodation models, such as Fudenberg and Tirole (1984). For an analysis of the timing of movie release dates see Krider and Weinberg (1998); also see McKenzie and Smirnov (2018). For empirical studies of strategic entry see Gil et al. (2019) (US drive-in cinemas), Schmidt-Dengler (2006) (MRI technology adoption in US hospitals) and Ellison and Ellison (2011) (pharmaceuticals with expiring patents).
} 
This paper draws on an extensive literature on innovation timing games. ${ }^{6}$ Our analysis of an irreversible investment decision with complete information and observable actions (closed-loop equilibria) follows Fudenberg and Tirole (1985), Dutta et al. (1995), Hoppe and Lehmann-Grube (2005) and Smirnov and Wait (2015). This framework has been used to study a range of applications. For example, Argenziano and Schmidt-Dengler (2012, 2013, 2014) adopt a variant of Fudenberg and Tirole (1985) to examine the order of market entry, clustering and delay. They show that with many potential entrants the most efficient firm need not be the first to enter the market and that delays are non-monotonic with the number of firms. In addition, they suggest a new justification for clustering of entry. Others have studied similar issues. Anderson et al. (2017) study delays and rushes into a market in a stopping game with a continuum of players. ${ }^{7}$

While we assume that previous actions of a rival are observable, an alternative approach to study innovation is to assume players' actions are unobservable as in Reinganum (1981a,b), where unobservable actions are equivalent to each firm being able to pre-commit. Reinganum shows that in the open-loop equilibria there will be diffusion in the sense that firms adopt the technology at different dates, even though all firms are ex ante identical. Similarly, Park and Smith (2005) develop an innovation game with unobservable actions that permits any firm (in terms of the order of entry) to receive the highest payoff. This allows for a war-of-attrition, with higher payoffs for late movers, a pre-emption game with higher payoffs for early movers, and a combination of both. An important point of comparison is that in our model firms use feedback rules to determine their strategy at any particular point in time; this means that they are unable to commit to their strategy at the beginning of the game.

Information also plays a key role in the players' entry strategies. Bloch et al. (2015) show that when two potential rivals are uncertain about their entry costs, competition leads to inefficient entry that is too early. Other authors consider inefficiencies in innovation when there is asymmetric information. For example, Bobtcheff and Mariotti (2012), Hendricks (1992) and Hopenhayn and Squintani

\footnotetext{
${ }^{6}$ See Hoppe (2002) or Van Long (2010, Chapter 5) for a survey of the literature. Further, Fudenberg and Tirole (1991) consider innovation when the firms make one irreversible decision (to enter) in a simple timing-game framework (see Sections 4.5 and 4.12).

${ }^{7}$ See also Riordan (1992) and Alipranti et al. (2011, 2015).
} 
(2011) assume that a firm's capability to innovate is private information. In these models, delay allows a firm to get better information about the potential innovation (its costs, value, and so on), but waiting runs the risk that a rival will innovate first, capturing the lion's share of the returns.

\section{The model}

Assume two firms $(i=1,2)$ are in a continuous-time stopping game starting at $t=0$ until some terminating time $T \in(0, \infty]$. Firm $i$ 's one-off decision to stop (that is, 'enter' the market) at $t_{i} \geq 0$ is irreversible and observable immediately by the other firm. The game ends when one of two firms has stopped/entered the market. The payoff to each firm depends on the stopping time. If the game ends with player $i$ stopping at time $t_{i}$, the payoffs of the leader and the follower are $L_{i}\left(t_{i}\right)$ and $F_{j}\left(t_{i}\right)$, respectively, where $i, j=1,2$ and $i \neq j$.

We make the following assumptions.

Assumption 1. Time is continuous in that it is 'discrete but with a grid that is infinitely fine'.

Assumption 2. Firms always choose to stop earlier rather than later in payoffequivalent situations.

Assumption 3. If more than one firm chooses to stop (enter) at exactly the same time, one of these firms is selected to stop (each with an ex ante probability of $\frac{1}{2}$ ).

Entry models in the literature adopt equivalent assumptions. Assumption 1 invokes Simon and Stinchcombe (1989) who show that under certain conditions a continuous-time strategy profile is the limit of a discrete-time game with increasingly fine time grids. It also replicates A1 of Hoppe and Lehmann-Grube (2005). ${ }^{8}$ Assumption 2, which is similar to A3 in Hoppe and Lehmann-Grube (2005), allows us to focus on just one (payoff-equivalent) equilibrium in the case of indifference between early and late entry. ${ }^{9}$ This simplifies our analysis so as to focus on the timing of entry rather than on issues of equilibrium selection.

\footnotetext{
${ }^{8}$ See Hoppe and Lehmann-Grube (2005), footnote 4 for a further discussion.

${ }^{9}$ Hoppe and Lehmann-Grube (2005) assume that if the follower is indifferent between two alternative entry times, it chooses the earliest time of entry.
} 
Assumption 3 - part of A3 in Hoppe and Lehmann-Grube (2005) and Assumption 5 in Dutta et al. (1995) - avoids potential coordination failures involving simultaneous entry. Given its importance, the intuition underlying this assumption warrants further discussion. In some situations, as a practical matter, if two firms try to enter the market at the same time there might be some capacity constraint or institutional requirement that prevents joint entry - consequently, one firm becomes the leader and the other firm is relegated to the role of second entrant. For instance, in a particular market there could be a bureaucratic rule that requires the leadership role be allocated to the firm that has the first email registered in a designated inbox. Even if both firms simultaneously send their messages, only one email can arrive first. As a consequence, with simultaneous moves, each firm has some probability of being the leader. Equivalent intuition applies to any (bureaucratic) tie-breaking rule that determines the winner in what seems to be a dead heat. Dutta et al. (1995) present a similar rationale for this assumption, suggesting there could be small random delays between when a decision is made and when a new technology is adopted, meaning that there is a positive probability that either firm will be first in the event of joint adoption. Here, Assumption 3 gives either firm an equal chance being first when there is simultaneous entry.

The following two assumptions ensure that the leader stops in finite time. ${ }^{10}$ The first element of this is that leaders' payoff functions reach their respective global maxima at a finite point in time; this means that both firms will not delay entry indefinitely. Dutta et al. (1995) (Assumption 3), Fudenberg and Tirole (1985) (Assumption 2(ii)) and Smirnov and Wait (2015) (Assumption 4) all make equivalent assumptions. Secondly, we assume that entering provides a higher payoff than each firm's respective outside option of zero, thus ensuring that our analysis is not unnecessarily complicated by having to consider whether one or both firms never enter the market. Again, this mirrors assumptions made previously in the literature; Assumption 4 in Dutta et al. (1995), Assumption 2(ii) in Fudenberg and Tirole (1985) and Assumption 5 in Smirnov and Wait (2015).

Assumption 4. There exists a finite $\widehat{T}_{i}<T$, which is the earliest time at which $L_{i}(t)$ attains its global maximum. Specifically, $L_{i}\left(\widehat{T}_{i}\right)>L(\tau) \forall \tau<\widehat{T}_{i}$, and

\footnotetext{
${ }^{10}$ When there is no ambiguity, we refer to payoffs as a function of $t$ rather than $t_{1}$.
} 
$L_{i}\left(\widehat{T}_{i}\right) \geq L(\tau) \forall \tau \geq \widehat{T}_{i}$ where $i=1,2$.

Assumption 5. Each firm's outside (non-entry) payoff is normalized to 0, and $L_{i}(t) \geq 0$ and $F_{i}(t) \geq 0 \quad i=1,2$.

Finally, we assume that the advantage of being second is non-increasing with the time of entry. This follows Hoppe and Lehmann-Grube (2005) who employ a similar assumption. In addition, this assumption incorporates the scenario studied in Argenziano and Schmidt-Dengler (2012, 2013, 2014), in which the follower payoff is constant with leader entry time. ${ }^{11}$

There are several possible explanations for why the follower's payoff would be non-increasing in the leader's entry time. If either cost fall or there is an improvement of the quality of the product with time, later entry by the leader could place the follower at a relative disadvantage; any delay in the initial entry time could help make the leader a stronger competitor, other things equal, hurting the follower. It is worth noting that this assumption is not crucial; rather, our key results hold in a more general environment. Assuming that the follower's payoff is non-increasing in the leader's time of entry, however, helps highlight the key economics insights of the model. We discuss this issue further in Section 3.2.3, in particular exploring the implications of allowing for non-monotonic follower payoffs.

To aid in exposition, we restrict our analysis to continuous leader and follower payoff functions. A detailed analysis solving entry games with discontinuous (but symmetric) payoffs can be found in Smirnov and Wait (2015).

This discussion is summarized in the following assumption.

Assumption 6. $L_{i}(t)$ is continuous, while $F_{i}(t)$ is continuous and non-increasing for $i=1,2$.

In summary, the first five assumptions are standard in the market-entry timing game literature with complete information and observable actions; see for example Smirnov and Wait (2015). Our last assumption is similar to Argenziano and

\footnotetext{
${ }^{11}$ It is worth noting that here we assume one potential innovation implemented by the market leader. In Argenziano and Schmidt-Dengler (2014), on the other hand, they model explicitly both firms entering the market. In equilibrium in their model entry by the follower always occurs at some later fixed date, resulting in a constant payoff for the follower.
} 
Schmidt-Dengler $(2012,2013,2014)$ and Hoppe and Lehmann-Grube (2005), however we allow for more generality in the structure of payoffs. Moreover, as noted above, we further investigate this assumption in Section 3.2.3.

To conclude this subsection, we outline two useful definitions. Firstly, provided $L_{i}(t)$ and $F_{i}(t)$ cross at least once, following Katz and Shapiro (1987), we define $\widetilde{T}_{i}$ to be the earliest time the payoff functions intersect.

Definition 1. If $L_{i}(t)$ and $F_{i}(t)$ intersect, $\widetilde{T}_{i} \leq T$, is the earliest time at which $L_{i}(t)=F_{i}(t)$.

Secondly, we also utilize the following definition.

Definition 2. Define $\bar{T}_{2} \leq \widetilde{T}_{2}$ to be the earliest time at which $L_{2}(t)$ attains its maximum for $t \in\left[0, \widetilde{T}_{2}\right]$.

\subsection{Equilibrium concept}

Following Fudenberg and Tirole (1985), we use subgame perfection. A history $h_{t}$ is defined as the knowledge of whether or not firm $i=1,2$ previously stopped at any time $\tau<t$, and if so when. A strategy of firm $i$, denoted by $\sigma_{i}\left(h_{t}\right)$, indicates at each history $h_{t}$ whether firm $i$ stops at $t\left(\sigma_{i}\left(h_{t}\right)=1\right)$ or does not stop at $t$ $\left(\sigma_{i}\left(h_{t}\right)=0\right)$. A strategy pair $\left(\sigma_{1}, \sigma_{2}\right)$ maps every history to an outcome, which is the minimum of stopping times $t_{1}$ and $t_{2}$. As usual, a strategy profile $\left(\sigma_{1}^{*}, \sigma_{2}^{*}\right)$ constitutes a subgame perfect equilibrium (SPE) if the strategies are sequentially rational after every history. Note here that with this representation we only need to specify the strategies when there has been no entry in the history of the game, because we assume that once one firm has entered, the game ends (Katz and Shapiro, 1987). This allows us, for ease of exposition, to refer to each firm's entry strategy as a function of time only, $\sigma_{i}(t)$.

\section{Characterization of equilibria}

In this section we first describe equilibria in the case of symmetric firms (Section 3.1), before exploring market entry when the firms potentially have different payoffs (Section 3.2). 


\subsection{Symmetric firms}

To outline a benchmark for the analysis that follows, first assume that both firms are the same in terms of their potential payoffs. The proposition below describes the method for determining the entry time of the leader in the symmetric case.

Proposition 1. [Smirnov and Wait (2015)] The equilibrium of the symmetric model is always unique. The first firm's stopping time $t^{*}$ is given by

$$
t^{*}=\min \arg \max _{t} \min [L(t), F(t)]
$$

As outlined in Smirnov and Wait (2015), this algorithm takes the minimum of the payoff functions for the leader and the follower, respectively. If the leader's payoff at the start of the game exceeds (or is equal to) the follower's payoff, given the follower's payoff is non-increasing, immediate entry $\left(t^{*}=0\right)$ maximizes the minimum of the two payoff functions (or is the earliest time to do so). In this case there is a first-mover advantage (or rents are equalized if $L(0)=F(0)$ ). Consider now the case when at the start of the game the follower's payoff exceeds that of the leader. Given that the follower's payoff is non-increasing, the first intersection between the two payoff functions $(\widetilde{T})$ is the only intersection that is economically relevant. If the leader's payoff is at its historical maximum at $\widetilde{T}$, entry occurs at this time (equalizing rents). This situation is illustrated in Figure 1(a). The bold line traces out the minimum of the leader and follower payoff functions. On the other hand, consider the situation when the leader's payoff at $\widetilde{T}$ is not at its historical maximum; see Figure 1(b). In this case there are two second-mover advantage equilibria in which one of the firms enters at $t^{*}$, while the other enjoys a higher payoff as the follower. Finally, when there is no intersection between $L(t)$ and $F(t)$, and $F(t)$ always exceeds $L(t)$, the leader enters at the time that $L(t)$ attains its global maximum $(\widehat{T})$. Again, there are two pure-strategy equilibria with a second-mover advantage, which entail either one of the firms acting as the market leader. 

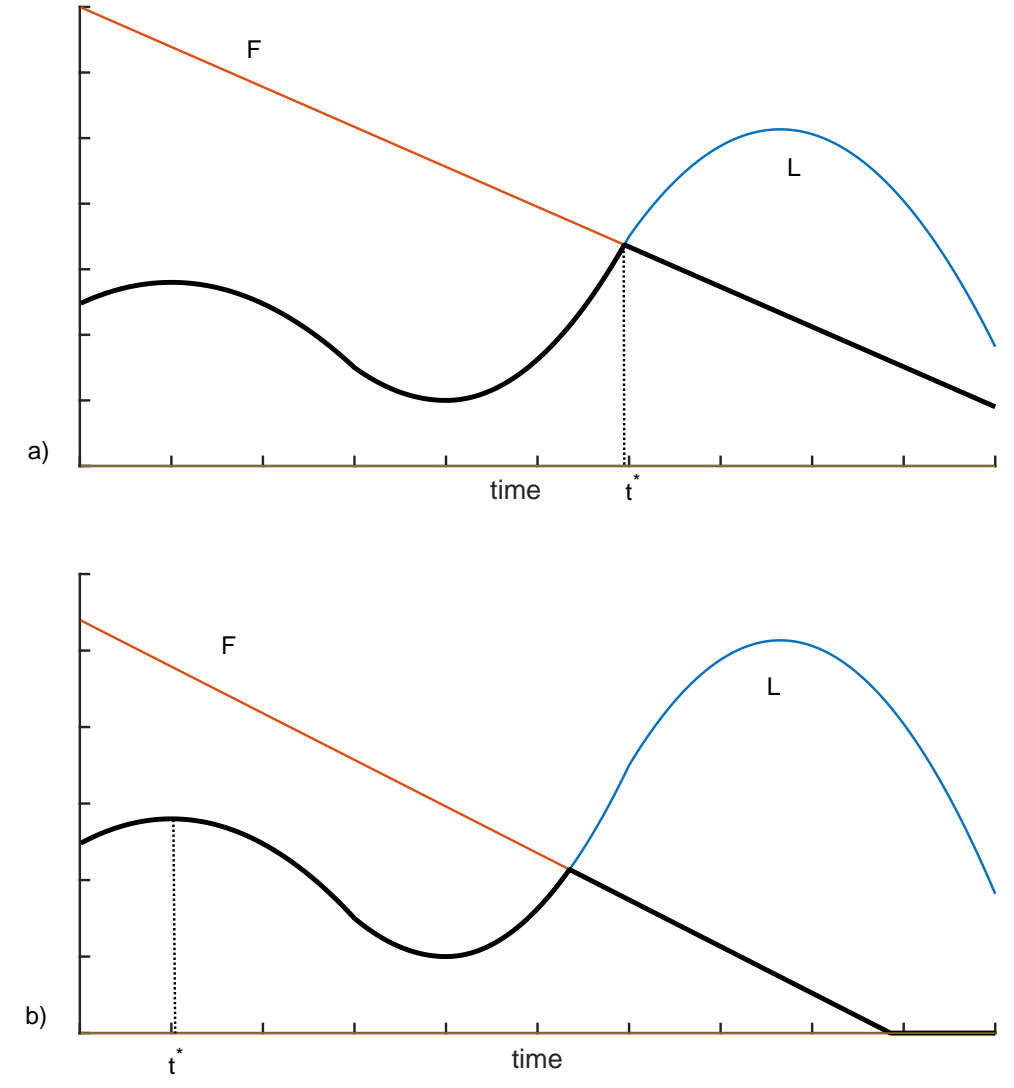

Figure 1: Preemption and second-mover advantage with symmetric firms 


\section{$3.2 \quad$ Asymmetric firms}

As noted previously, firms are more often than not different from one another. In this section we develop a method of determining the leader's entry time in all pure-strategy SPE, allowing for asymmetric payoff functions. Firstly, to find the pure-strategy SPE we note that any equilibrium with player i entering at time $t_{i}$ must satisfy two necessary conditions:

Condition 1. No preemption by the leader $i(N P L): L_{i}\left(t_{i}\right)>L_{i}(\tau), \forall \tau \in\left(0, t_{i}\right)$.

Condition 2. No preemption by the follower $j(N P F): F_{j}\left(t_{i}\right)>L_{j}(\tau), \quad \forall \tau \in$ $\left(0, t_{i}\right)$ and $F_{j}\left(t_{i}\right) \geq L_{j}\left(t_{i}\right)$.

If the $N P L$ does not hold, the leader (player i) will deviate by entering earlier. Similarly, the NPF must hold in any SPE, otherwise the follower (player $\mathrm{j}$ ) has an incentive to preempt and enter slightly earlier than the leader, as in Fudenberg and Tirole (1985). ${ }^{12}$ Even if these conditions hold, they do not in of themselves guarantee that a specific entry time is part of an SPE, because both only compare payoffs at a particular time relative to their historic values. These conditions, by definition, do not make any comparisons with future potential payoffs. Of course, such a consideration is necessary when determining any SPE.

To solve for the leader's entry time, let us eliminate all points that do not satisfy either of these conditions (the NPL and the NPF) by constructing sets $A_{1}\left(t^{\prime}, t^{\prime \prime}\right)$ and $A_{2}\left(t^{\prime}, t^{\prime \prime}\right)$. For each firm $i \in\{1,2\}, j \neq i$ and $T \geq t^{\prime \prime}>t^{\prime} \geq 0$, define the following set:

$A_{i}\left(t^{\prime}, t^{\prime \prime}\right)=\left\{t \in\left(t^{\prime}, t^{\prime \prime}\right] \mid L_{i}(t)>L_{i}(\tau) \& F_{j}(t)>L_{j}(\tau) \forall \tau \in\left(t^{\prime}, t\right) \& F_{j}(t) \geq L_{j}(t)\right\}$

By definition, a point belongs to set $A_{i}\left(t^{\prime}, t^{\prime \prime}\right)$ if it satisfies both $N P L$ and $N P F$. By way of comparison, to solve the symmetric-player entry game Smirnov and Wait (2015) construct one set that is applicable to both firms. Here, asymmetry requires the construction of a set $A_{i}($.$) for each firm and for any truncated game$ played on interval $\left[t^{\prime}, t^{\prime \prime}\right]$.

\footnotetext{
${ }^{12}$ Argenziano and Schmidt-Dengler (2014) adopt similar conditions, which they refer to as the Leader Preemption Constraint and the Follower Preemption Constraint.
} 
For each firm $i \in\{1,2\}$ define the following time

$$
t_{i}^{*}=\left\{\begin{array}{ccc}
\arg \max _{t} A_{i}(0, T) & \text { when } & A_{i}(0, T) \neq \emptyset \\
0 & \text { when } & A_{i}(0, T)=\emptyset .
\end{array}\right.
$$

In addition, assume without loss of generality that $t_{1}^{*} \geq t_{2}^{*}$. Moreover, for the truncated game played on $\left[0, t_{2}^{*}\right]$ define the following time

$$
t_{1}^{* *}=\left\{\begin{array}{ccc}
\arg \max A_{1}\left(0, t_{2}^{*}\right) & \text { when } & A_{1}\left(0, t_{2}^{*}\right) \neq \emptyset, \\
0 & \text { when } & A_{1}\left(0, t_{2}^{*}\right)=\emptyset .
\end{array}\right.
$$

Note that by construction $t_{i}^{*}$ and $t_{1}^{* *}$ are always unique. $t_{i}^{*}$ describes the optimal entry time if player $i$ has to be the leader, while $t_{1}^{* *}$ describes the optimal entry time if player 1 has to preempt player 2 , who is expected to enter at $t_{2}^{*}$.

\subsubsection{Preemption and second-mover advantage equilibria}

In this section we explore situations in which the familiar preemption and secondmover advantage equilibria arise in the case with asymmetric firms. To do so consider equilibria when $A_{1}\left(t_{1}^{*}, T\right)=\emptyset$; the entry decision at $t_{1}^{*}$ is not affected by historical payoffs. The following proposition summarises all potential equilibria in this case.

Proposition 2. Consider the SPE of the two-player asymmetric timing game when $A_{1}\left(t_{1}^{*}, T\right)=\emptyset$. If

1. $L_{1}\left(t_{1}^{*}\right)>F_{1}\left(t_{1}^{*}\right)$, the SPE involves firm 1 entering at $t=t_{1}^{*}$;

2. $L_{1}\left(t_{1}^{*}\right) \leq F_{1}\left(t_{1}^{*}\right)$, there are two $S P E$, one with firm 1 entering at $t=t_{1}^{*}$ and the other with firm 2 entering at $t=t_{2}^{*}$.

Proof: See Appendix B.

When $L_{1}\left(t_{1}^{*}\right)>F_{1}\left(t_{1}^{*}\right)$, as in Proposition 2(1), there are two possible cases. As explained below, both involve firm 1 playing the role of a market leader. Moreover, in essence both are a small perturbation of the scenario presented in Figure 1(a) with symmetric players. 

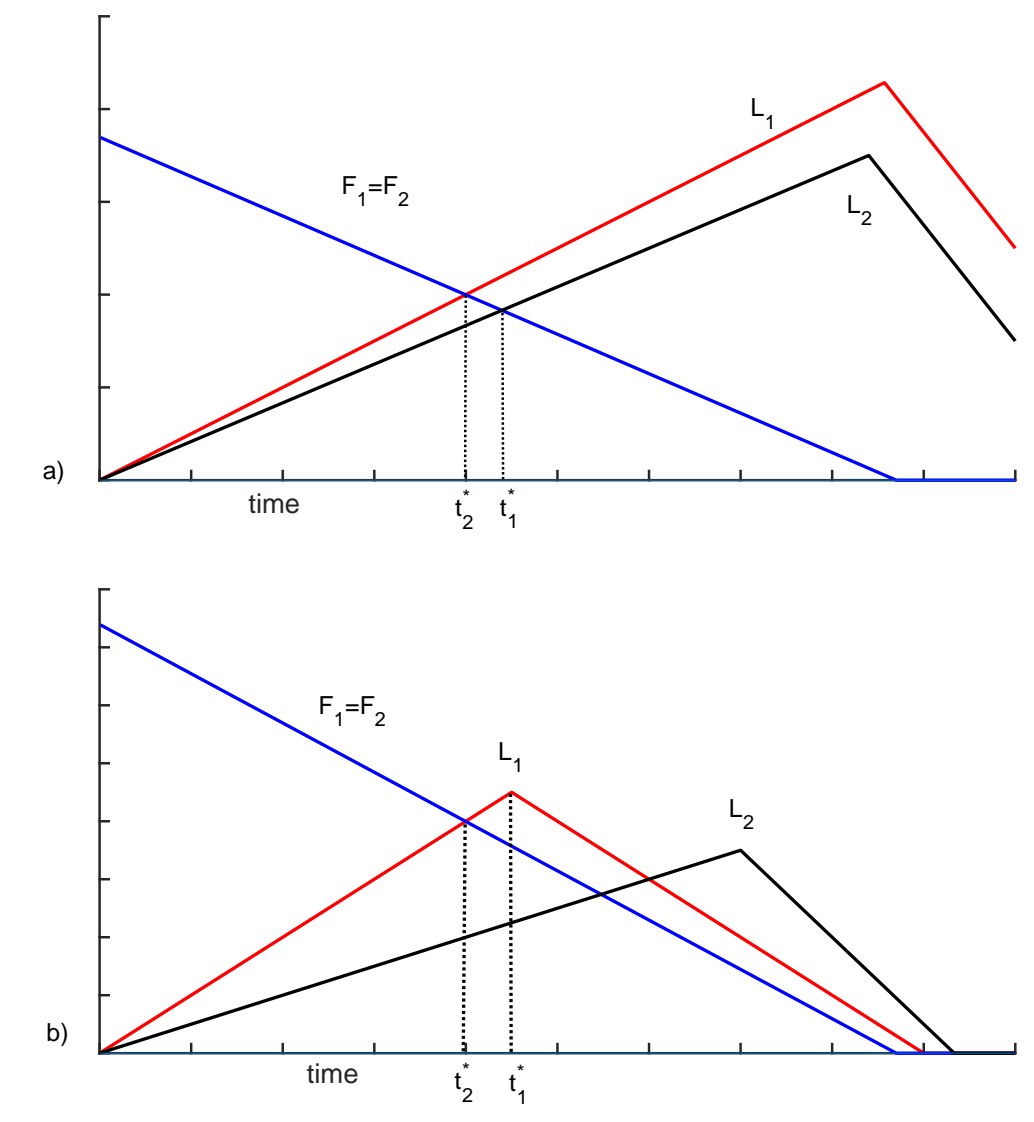

Figure 2: $A_{1}\left(t_{1}^{*}, T\right)=\emptyset$ and $L_{1}\left(t_{1}^{*}\right)>F_{1}\left(t_{1}^{*}\right)$ : entry occurs at $t_{1}^{*}$ 
The first possibility is illustrated in Figure $2(\mathrm{a}) .{ }^{13}$ In this case the global maxima for both leader payoff functions are at an entry time after $t_{1}^{*}$. Given the incentive to preempt, entry will occur before either of $\widehat{T}_{i}$. Rather, firm 1 enters at $t_{1}^{*}=\widetilde{T}_{2}$ (where $L_{2}$ crosses $F_{2}$ ) so as to just preempt entry by its rival.

Now consider the case when $\widetilde{T}_{1}<\widehat{T}_{1}<\widetilde{T}_{2}$, as illustrated in Figure 2(b). Firm 1 will enter at the time that maximizes its leader payoff, $\widehat{T}_{1}$. Again there is a unique time of entry $-t_{1}^{*}$ - but in this case firm 1 enters at its stand-alone time unlike the case above where strategic interactions lead to earlier entry. The strategies firms adopt in Proposition 2(1) are:

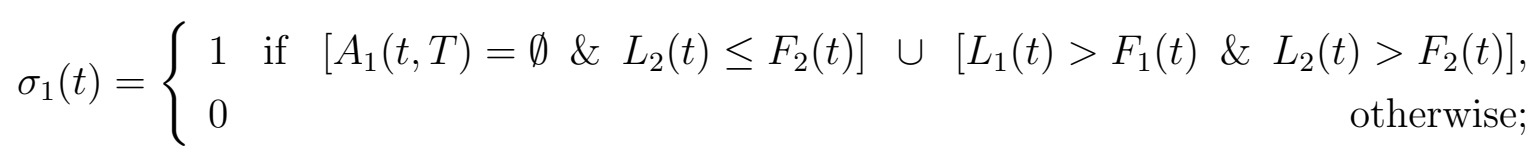

$$
\sigma_{2}(t)=\left\{\begin{array}{lrr}
1 & \text { if } & L_{2}(t)>F_{2}(t) \\
0 & \text { otherwise. }
\end{array}\right.
$$

These strategies require that firm 1 enters in two distinct situations. Firstly, firm 1 opt to be the leader when it has no further incentive to wait in the hope of a higher return later $\left(A_{1}\left(t_{1}^{*}, T\right)=\emptyset\right)$ and firm 2 prefers to be a follower, as $L_{2}(t) \leq F_{2}(t)$. The second situation resembles the classic preemption game outlined in Fudenberg and Tirole (1985), as both firms prefer to be a leader rather than a follower when both $L_{1}(t)>F_{1}(t) \& L_{2}(t)>F_{2}(t)$. On the other hand, considering the strategy of firm 2 , it will only enter the market at $t$ if doing so dominates waiting. This holds when $L_{2}(t)>F_{2}(t)$, remembering that $F_{2}(t)$ is a non-increasing function.

When $L_{1}\left(t_{1}^{*}\right) \leq F_{1}\left(t_{1}^{*}\right)$, as in Proposition 2(2), there are two distinct scenarios. First, there is the possibility that the earliest intersection between either leader and follower payoff curve occurs at the same time - that is $\widetilde{T}_{1}=\widetilde{T}_{2}$, as illustrated in Figure 3(a). As in the two scenarios discussed above, this is also a small perturbation of the symmetric case in Figure 1(a). The incentive to preempt ensures that entry occurs at the time of first intersection of each $L_{i}$ and $F_{i}$; there are in fact two equilibria with either firm entering at $t=\widetilde{T}_{1}=\widetilde{T}_{2}$.

The second scenario is illustrated in the example shown in Figure 3(b). Note

\footnotetext{
${ }^{13}$ Both in this example and in most of the examples that follow we make the simplification that $F_{1}=F_{2}$ for illustrative purposes.
} 

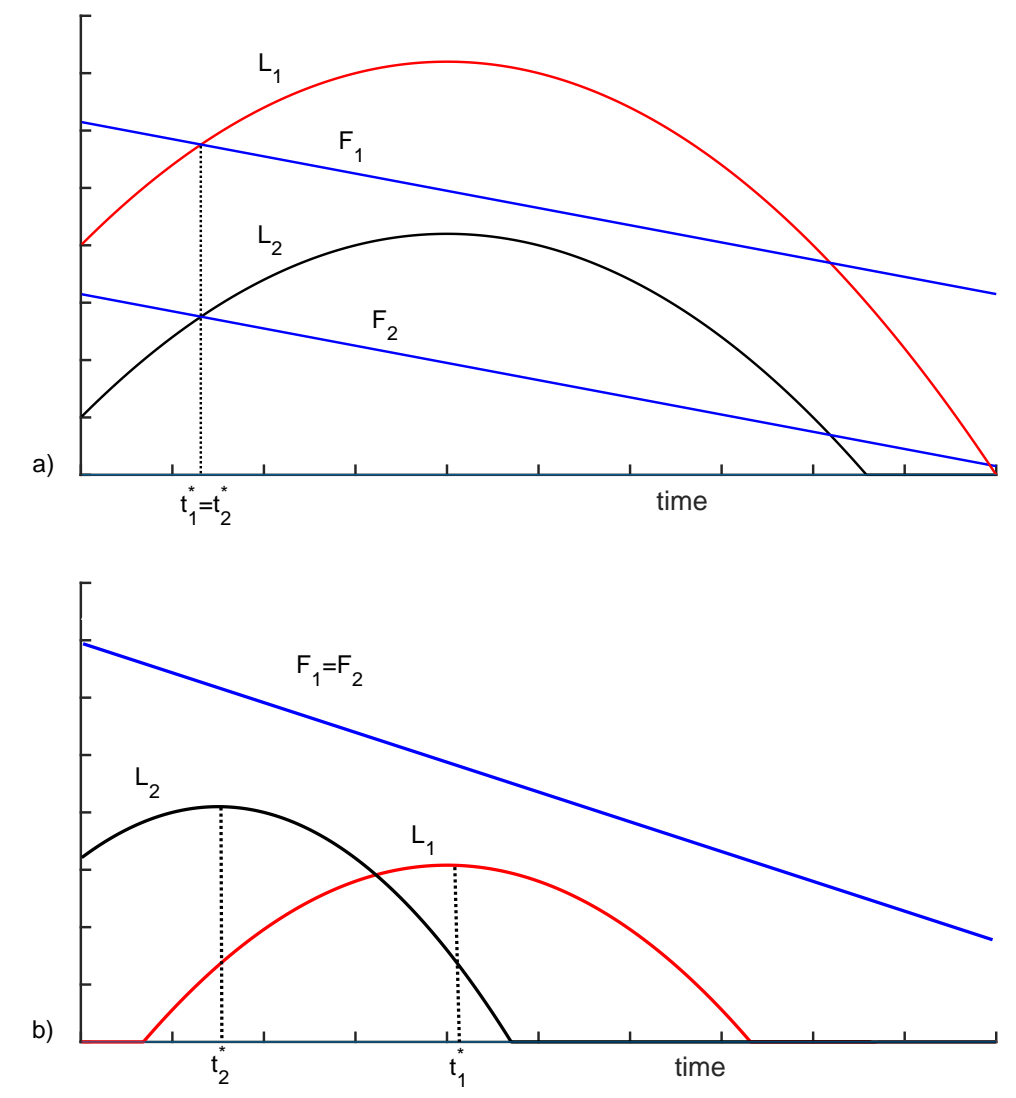

Figure 3: $A_{1}\left(t_{1}^{*}, T\right)=\emptyset$ and $L_{1}\left(t_{1}^{*}\right) \leq F_{1}\left(t_{1}^{*}\right)$ : entry occurs at either $t_{1}^{*}$ or $t_{2}^{*}$ 
that $L_{1}\left(t_{1}^{*}\right) \leq F_{1}\left(t_{1}^{*}\right)$. Moreover, $A_{1}\left(t_{1}^{*}, T\right)=\emptyset$ guarantees that $L_{2}\left(t_{2}^{*}\right)<F_{2}\left(t_{2}^{*}\right)$. In this case there are two SPE in which both firms prefer to be the follower. Specifically, in each of these equilibria one of the firms enters when they attain their highest leader payoffs $\widehat{T}_{i}$ (and the other firm always waits, unless entering strictly dominates waiting). Note that this example is a small perturbation of the symmetric-players case illustrated in Figure 1(b).

For SPE in Proposition 2(2) where firm i is the leader and firm $\mathrm{j}$ is the follower, the firms' strategies in each of the SPE are:

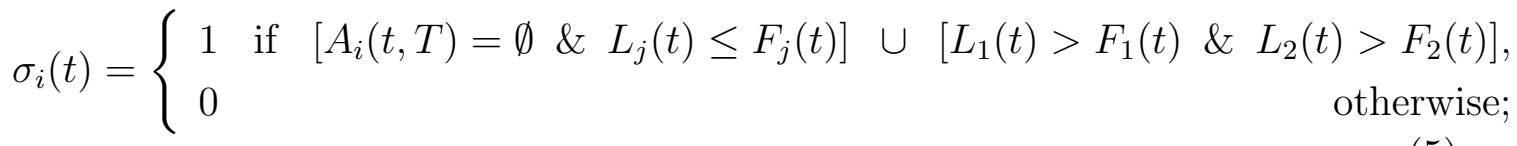

$$
\begin{aligned}
& \sigma_{j}(t)=\left\{\begin{array}{lrr}
1 & \text { if } & L_{j}(t)>F_{j}(t) \\
0 & \text { otherwise. }
\end{array}\right.
\end{aligned}
$$

These strategies generalize those outlined earlier for Proposition 2(1) above and apply to all equilibria discussed so far. Consider the equilibrium when firm 2 enters, so it plays the role of firm $i$. In this case, firm 2's strategy is to wait until its payoff (as a leader) is maximized, and then to enter at this time or whenever $A_{2}(t, T)=\emptyset \& L_{1}(t) \leq F_{1}(t)$. If, somehow, a preemption subgame is reached in which $L_{1}(t)>F_{1}(t)$ and $L_{2}(t)>F_{2}(t)$, firm 2 would enter as entering dominates waiting. Conversely, firm 1 plays the role of follower in this equilibrium. It will wait in any subgame, unless its leader payoff exceeds the return from being a follower.

It is worth noting that the equilibria outlined in this Section mimic the equilibria discussed in the existing entry-timing literature with symmetric players, notably preemption and second-mover advantage equilibria. Now we turn our attention to novel history-dependent equilibria.

\subsubsection{Blocking equilibria}

In this section we consider new equilibria not possible in the symmetric case when entry decisions could be affected by historical payoffs. These cases arise when $A_{1}\left(t_{1}^{*}, T\right) \neq \emptyset$. We characterize all of these SPE in the following proposition. 
a)

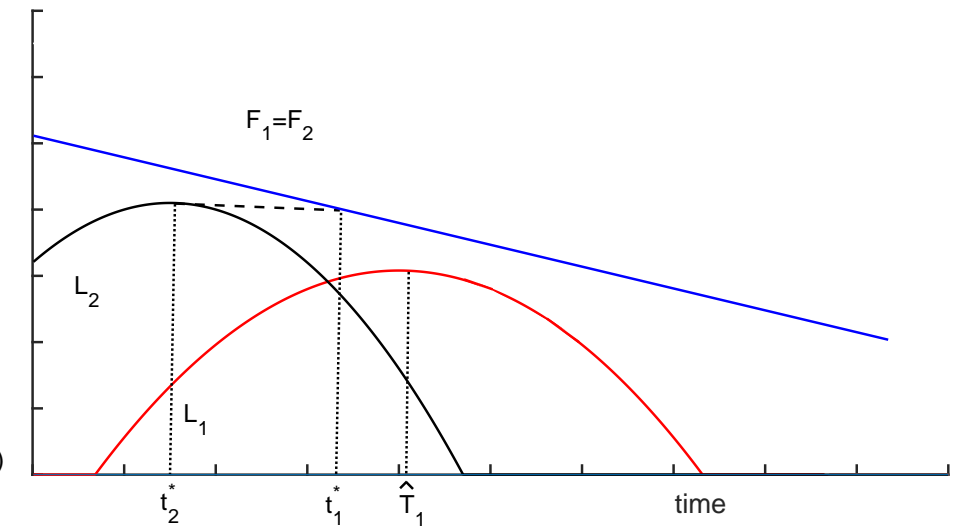

b)

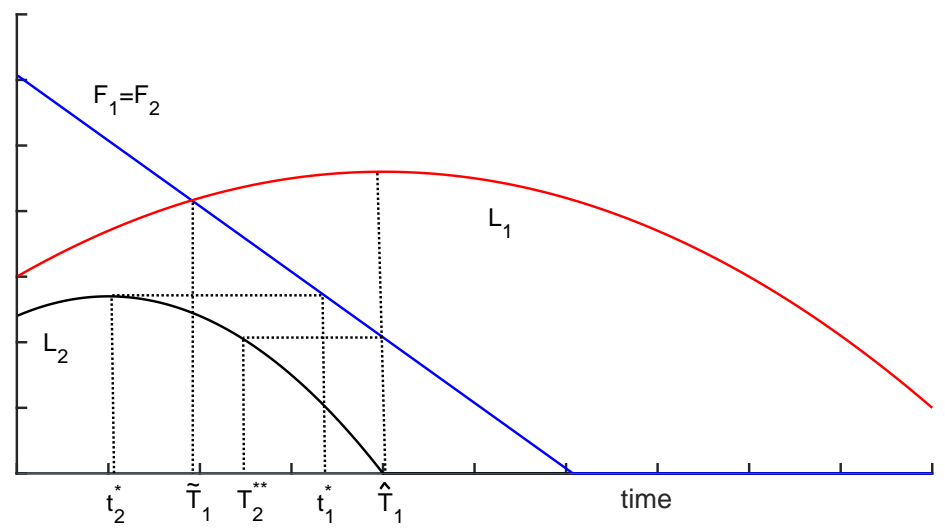

Figure 4: Example of SPE in Proposition 3(2), when $A_{1}\left(t_{1}^{*}, T\right) \neq \emptyset$ and $t_{2}^{*}=\widehat{T}_{2}$ : firm 2 is the market leader, entering at $t_{2}^{*}$

Proposition 3. Consider the SPE of the two-player asymmetric timing game when $A_{1}\left(t_{1}^{*}, T\right) \neq \emptyset$. If

1. $t_{2}^{*}<\bar{T}_{2}$ and $A_{2}\left(t_{2}^{*}, t_{1}^{*}\right)=\emptyset$, there is no SPE;

2. $t_{2}^{*}=\bar{T}_{2}$ and $A_{2}\left(t_{2}^{*}, t_{1}^{*}\right)=\emptyset$, there is a unique SPE with firm 2 entering at $t=t_{2}^{*}$;

3. $A_{2}\left(t_{2}^{*}, t_{1}^{*}\right) \neq \emptyset$, there is a unique SPE involving firm 1 entering at $t=t_{1}^{* *}$.

Proof: See Appendix B.

To garner some intuition for these results, with the help of Figure 4, first consider Proposition 3(2). As illustrated in the top panel of the Figure, if the 


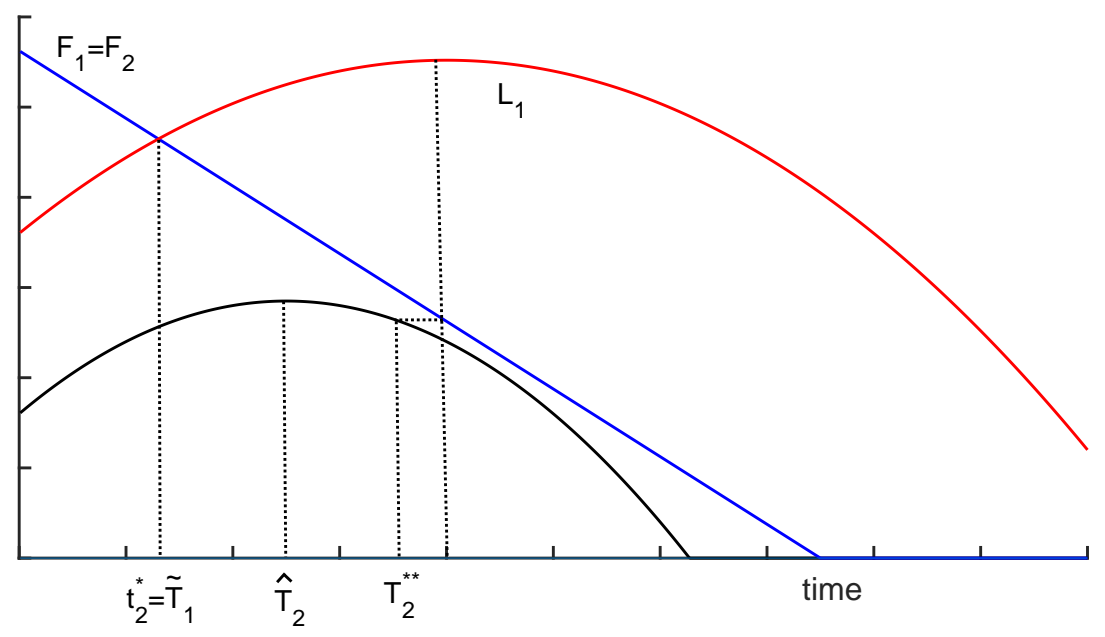

Figure 5: Example of no pure-strategy SPE in Proposition 3(1), when $A_{1}\left(t_{1}^{*}, T\right) \neq \emptyset$ and $t_{2}^{*}<\widehat{T}_{2}$

game reaches $t_{1}^{*}$ without entry, firm 1 would not enter at this time; rather it has an incentive to wait and enter at $\widehat{T}_{1}$. Understanding firm 1 's incentive, as $F_{2}\left(\widehat{T}_{1}\right)<$ $L_{2}\left(t_{2}^{*}\right)$, firm 2 has an incentive to block its rival by entering at $t_{2}^{*} \cdot{ }^{14}$ Consequently, there is a unique equilibrium with firm 2 entering at $t_{2}^{*}$. The strategies adopted in this case are outlined in equations (5) - (6) with firm 2 playing the role of player $i$.

Note that this example satisfies the condition $L_{1}\left(t_{1}^{*}\right)<F_{1}\left(t_{1}^{*}\right)$. However, in general any sign between $L_{1}\left(t_{1}^{*}\right)$ and $F_{1}\left(t_{1}^{*}\right)$ is possible. To illustrate this, consider the example shown in Figure 4(b), in which firm 1 has a leader advantage (after $\left.\widetilde{T}_{1}\right)$ whereas firm 2 prefers to wait. This example is equivalent to the example presented in Figure 2C in Katz and Shapiro (1987). Employing the same logic as in the example in Figure 4(a), if the game were to reach $t_{1}^{*}$ without entry, firm 1 would have an incentive to wait and only enter at $\widehat{T}_{1}$. At this time in the game, there is no credible way firm 2 can prevent firm 1 from waiting, as firm 2's follower payoff exceeds its return as a leader. Anticipating this, firm 2 has an incentive to block its rival; there is a unique equilibrium with firm 2 entering at $t_{2}^{*}$ (its historical maximum payoff as leader). As noted by Katz and Shapiro (1987), however, there is a complication that there does not exist an equilibrium with pure strategies for

\footnotetext{
${ }^{14}$ In Figure $4(\mathrm{a}) A_{1}(0, T)=\left(0, t_{1}^{*}\right]$, where $t_{1}^{*}$ is determined by historical maximum of $L_{2}$. On the other hand, $A_{2}(0, T)=\left(0, t_{2}^{*}\right]$.
} 
a truncated subgame on $\left[\widetilde{T}_{1}, T_{2}^{* *}\right]$. This issue is discussed in Katz and Shapiro (1987), in footnote 15. One solution is to resort to mixed strategies. Another possibility is to augment the equilibrium concept so as to ignore subgames with dominated strategies. This caveat ensures entry by firm 2 at $t_{2}^{*}$, regardless of the strategies adopted by both firms after this time. ${ }^{15}$

This issue of non-existence of equilibria is exacerbated further in Proposition 3(1), illustrated in Figure 5. In this case there is no pure-strategy SPE. Critical to this non-existence outcome is that $t_{2}^{*}<\widehat{T}_{2}$. The intuition for this result is as follows. In this scenario, recall that $A_{1}\left(t_{1}^{*}, T\right) \neq \emptyset$. This means that if the game reaches $t_{1}^{*}$ without entry, firm 1 will have an incentive to delay entry further. Anticipating this, firm 2 would consider blocking its rival by entering earlier; a candidate for blocking entry would be at a time at which its leader payoff is maximized, $\widehat{T}_{2}$. But in this example $t_{2}^{*}<\widehat{T}_{2}$, which raises the problem of existence as there is no pure strategy equilibrium for $\left[\widetilde{T}_{1}, T_{2}^{* *}\right]$. At $\widehat{T}_{2}$ firm 1 's best response to entry by firm 2 is to preempt, as $L_{1}\left(\widehat{T}_{2}\right)>F_{1}\left(\widehat{T}_{2}\right)$. Firm 2 would prefer to follow if firm 1 enters, but if it does so, firm 1 would also have an incentive to wait, as its leader payoff is increasing at this time. Hence, there is no combination of best-response pure strategies, as formally captured by Proposition 3(1).

Before we proceed it is worth noting that in all cases we considered so far, (if equilibria exist) entry by the market leader always occurs at $t_{1}^{*}$ or at $t_{2}^{*}$, or at both times (which could of course coincide). While not ensuring uniqueness, it does indicate that at most there are two entry times feasible in equilibrium.

Now we turn our attention to Proposition 3(3). Let us highlight the intuition of this case with the assistance of the example illustrated in Figure $6 .{ }^{16}$ Firstly, as shown in Figure 6 , note that $A_{1}(0, T)=\left(0, t_{1}^{* *}\right] \cup\left(t_{3}, t_{1}^{*}\right]$. On the other hand, $A_{2}(0, T)=\left(0, t_{2}^{*}\right]$. To determine the equilibrium entry time, we iterate backwards from the latest possible candidate entry date. If game has reached $t_{1}^{*}$ without entry, firm 1 would rather wait and enter later at $\widetilde{T}_{2}$ than to lead at $t_{1}^{*}$. Anticipating firm 1's incentive to wait at $t_{1}^{*}$, firm 2 would be tempted to preemptively block this outcome; as $A_{2}(0, T)=\left(0, t_{2}^{*}\right]$ a candidate entry time is $t_{2}^{*}$. However, if the

\footnotetext{
${ }^{15}$ Regarding the statement of Proposition 3, we treat this scenario as if the equilibrium exists and either mixed strategies can be used or subgames with dominates strategies can be ignored.

${ }^{16}$ Micro-foundations for this example are presented in Appendix A.
} 


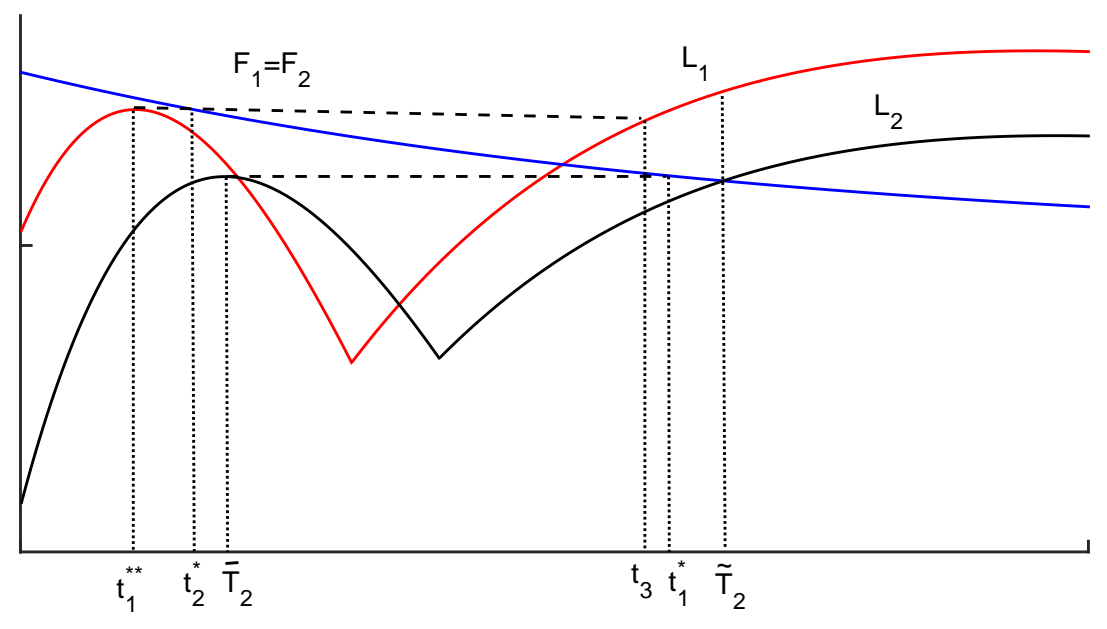

Figure 6: Example of blocking equilibrium with two iterations in Proposition 3(3), when $A_{1}\left(t_{1}^{*}, T\right) \neq \emptyset$ and $A_{2}\left(t_{2}^{*}, t_{1}^{*}\right) \neq \emptyset$

game reaches $t_{2}^{*}$, both firms will have an incentive to wait as firm 2's leader payoff is increasing and firm 1 has a higher payoff as a follower. In fact, rather than entering at $t_{2}^{*}$, firm 2 would prefer to enter at $\bar{T}_{2}$. Of course, anticipating its fate, firm 1 will block this eventuality by entering even earlier at $t_{1}^{* *}$, ensuring a payoff of $L_{1}\left(t_{1}^{* *}\right)$ which exceeds its follower payoff at $\bar{T}_{2}$.

For SPE in Proposition 3(3) the firms' strategies are:

$$
\sigma_{1}(t)=\left\{\begin{array}{lr}
1 & \text { if } \quad\left[A_{1}\left(t, t_{2}^{*}\right)=\emptyset \& L_{1}(t)>F_{1}\left(\bar{T}_{2}\right)\right] \cup\left[L_{1}(t)>F_{1}(t)\right] \\
0 & \text { otherwise }
\end{array}\right.
$$

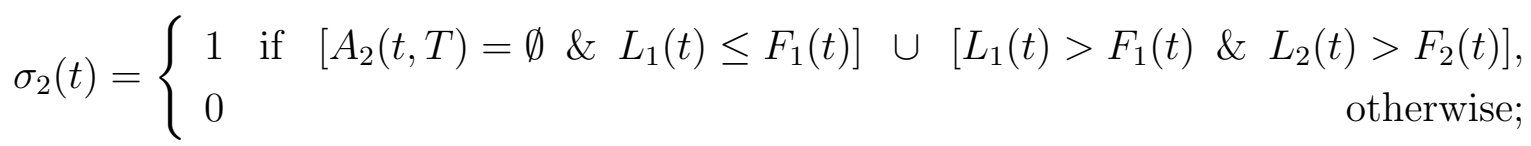

These strategies amend those outlined in equations (5) - (6), with firm 2 playing the role of player $i$, to include the requirement that firm 1 enters to block firm 2's entry at $\bar{T}_{2}$ when $A_{1}\left(t, t_{2}^{*}\right)=\emptyset$ and $L_{1}(t)>F_{1}\left(\bar{T}_{2}\right)$.

In summary, firm 1 comes into the market early to block firm 2's strategic entry plans, which are in of themselves an attempt to preemptively block firm 1's future leadership intentions. That is, anticipating that it will be preempted by firm 2 
at $\bar{T}_{2}$, firm 1 blocks this preemption by entering even earlier, in this case at $t_{1}^{* *}$. This is another version of a blocking equilibrium, this time with two iterations. As noted in the Introduction, this could relate to a firm prematurely launching a new smartphone, or cobbling together an updated release in an attempt to block a rival's future entry that would have, in its own right, a preemptive market entry. This blocking equilibrium is only possible with heterogenous firms and when equilibrium strategies are influenced by historic payoffs. This example also illustrates that in the general case covered by the Proposition 3, entry times in equilibrium can differ from $t_{1}^{*}$ and $t_{2}^{*}$.

\subsubsection{Continuous version of centipede game}

So far we have assumed that the follower payoff functions are non-increasing with the time of entry by the leader. Even in that case, a blocking equilibrium with two iterations is possible. Here we show that any arbitrary number of these strategic leapfrogging iterations are possible in the general case. To see this, consider the following continuous (market-entry) version of the centipede game illustrated in Figure 7. Note that while there is a positive trend in the payoffs to each firm, they experience several peaks and troughs along the way. These mountainous looking payoffs could represent the seasonally changing payoffs associated with new versions of two smartphones as they become available. Important also in this example is that the local peak for one firm roughly corresponds to a time of a local trough for its rival. In the Figure, firm 1's potential payoffs are shown in panel (a) with the top line being its follower payoff $F_{1}$ and the bottom line its leader payoff $L_{1}$, whilst panel (b) shows the follower and leader payoffs for firm 2 . Consider the equilibrium outcome of this centipede entry model. As with the previous blocking equilibria, if the game reaches $t_{1}^{*}$ without entry, firm 1 would not continue waiting until $t_{1}^{1}$, as its leader payoff is increasing and there is no credible threat of entry by firm 2. This is a situation that firm 2 wishes to avoid, so it would be willing to enter at $t_{2}^{1}$ to block firm 1's opportunistic actions after this time. But, given the non-monotonic nature of the payoffs, this entry time provides firm 1 with a lower follower payoff than it could have got as a leader previously. Moreover, entry by firm 2 at $t_{2}^{*}$ is not credible, because firm 2's leader payoff is increasing at this 


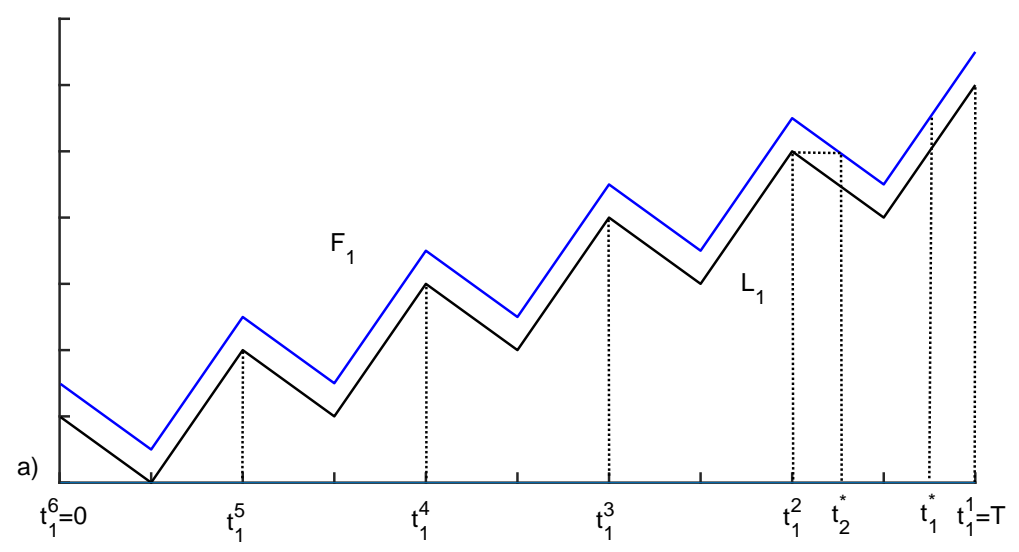

b)

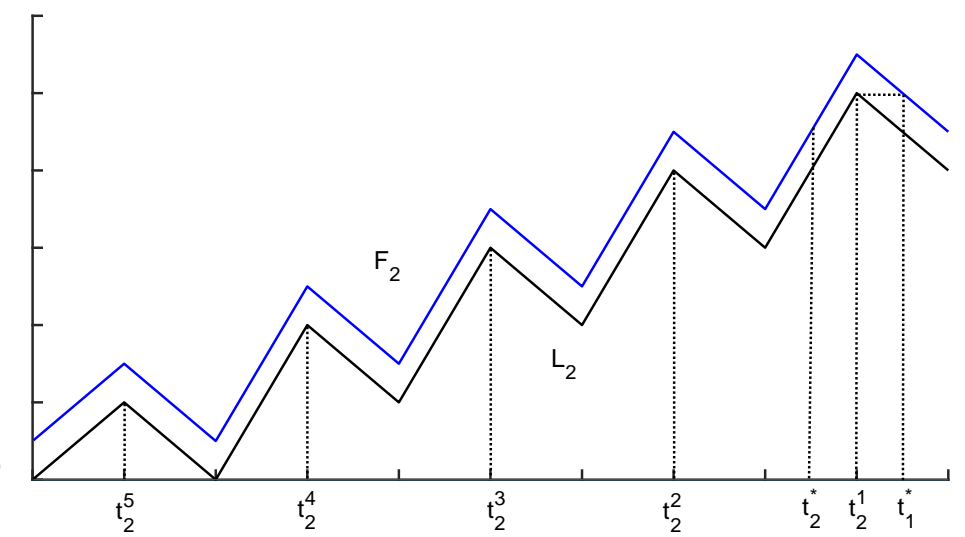

Figure 7: Continuous version of the centipede game

time. To gazump this, firm 1 will come in at $t_{1}^{2}$, if there had been no entry prior. This blocking process of iterating backwards moves through all of the peaks (and corresponding rival troughs) - before entry in equilibrium occurs immediately at $t_{1}^{6}=0$. The intuition for blocking is the same as above - to avoid a lower future payoff a firm will enter earlier, a move which itself induces even earlier entry by its rival, and so on. In this game, the payoffs to the two firms need not be equalized, as in the classic preemption equilibrium. Moreover, entry here is inefficient - both firms could be made better off if they could wait and enter later.

Also note that, more generally, our solution technique can be applied to the case when both leader and follower payoff functions take on any continuous shape. While it is tedious to express the entry strategies and existence of equilibria in the general case, the intuition of our solution algorithm from the base case holds and 
can be used to solve for the entry equilibrium in any specific game desired.

\section{Concluding comments}

The decision when to launch a new product or production process is a critical question for many firms; it can help determine profit, firm survival and the shape of markets. More generally, it drives economic development. Given its importance, innovation has received a great deal of attention from economists, such as in Fudenberg and Tiroles' seminal work. Getting the timing of innovation right is difficult enough for any firm, let alone when it has a rival breathing down its neck. In this paper we examine innovation as a market entry game with duopoly rivals in a very general framework; our main model only assumes that payoffs are continuous and that follower payoffs are non-increasing in entry time of the leader. Most importantly, inspired by Katz and Shapiro (1987), we allow for asymmetries between firms. This incorporates the situation when firms have different capabilities or technologies. Moreover, allowing for non-monotonic leader payoffs captures situations where multiple technologies or products become available for adoption at different times.

Adapting the solution method of Smirnov and Wait (2015) to allow for asymmetric payoffs, we solve for all pure strategy subgame perfect equilibria, characterized by the time of the leader's market entry. It turns out that there can be zero, one or at most two pure strategy equilibria. In some cases, we see preemption or a second-mover advantage in the asymmetric game, familiar from the symmetric case. But other outcomes are also possible. Notably, in our blocking equilibrium we show that the threat of future entry can lead to an iterative process in which each firm would enter earlier, so as to block the future entry plans of their rival. This results in a very early entry by the market leader, and is suggestive of immature product launches by firms attempting to avoid lower payoffs anticipated if their rival becomes the market leader in the future. Importantly, our blocking equilibria differ from the preemption equilibrium in Fudenberg and Tirole (1985) in that rents do not have to be equalized. This analysis also differs from Katz and Shapiro (1987) in that our blocking equilibrium can have any number of backwards iterations, given the shape of potential payoff functions, whereas in their 
model there can be at most one blocking entry due to their implicit assumption of concave payoffs.

The generality of our solution method is demonstrated by our application to the entry game with non-monotonic follower (and leader) payoffs. Moreover, in this case, the iterative incentives to block each other's product launch leads to starkly inefficient early entry in a continuous version of the centipede game.

Finally, two further caveats are worth noting. Firstly, we have assumed continuous payoffs, leaving the analysis of discontinuities in the (symmetric) entry game to Smirnov and Wait (2015). If this assumption is relaxed, additional equilibria in pure strategies corresponding to points of discontinuities are possible. Secondly, we have effectively ruled out the situations where both pairs of leader and follower curves intersect at the same points more than once and these intersections correspond to both leaders' respective historical maxima. These points of intersection match rent-equalizing equilibria, that for the (symmetric) entry game were analyzed in Smirnov and Wait (2015).

\section{Appendix A - Example of innovation with two decreasing-cost innovation choices}

To provide some further intuition for the main results in the paper, and to allow for a closer comparison with the previous literature, we construct the following modification of Katz and Shapiro (1987). Essentially, we augment their example to allow for more than one potential innovation that firms can put into practice. As noted in the introduction, firms are often faced with the choice between two or more alternative technologies. Examples of competing technologies for tablets, phone handsets and computer hardware come to mind, but a similar choice often has to be made when considering adopting cost-reducing technologies. Each technology will typically come with its own advantages and, moreover, the relative advantages of a given technology can change over time. Our framework is general enough to capture all of these scenarios.

Here, we completely characterize all SPE of this two-player innovation game using the algorithm outlined in this paper. By doing so, we show how an augmented 
example of Katz and Shapiro (1987) can provide micro-foundations for the blocking equilibrium with two iterations, as illustrated in Figure 6 and discussed above.

Consider the case when two firms are contemplating when to upgrade to a new technology, which they can implement at some time $t_{i} \in[0, \infty)$ for $i=1,2$. Each firm can choose to implement one of the two options $k=1,2$ available. For each firm, the old (null) technology generates a flow of profit normalized to zero; that is, $\pi_{i}^{0}=0, i=1,2$. After adoption, the new technology $k$ affords firm $i$ a flow of profit $\pi_{i k}^{i}>0$. Furthermore, subsequent to firm $i$ adopting technology $k$, firm $j$ 's earns a flow profits of $\pi_{j k}^{i}>0$. Finally, after firm $i$ 's adoption of $k$, industry profits are given by $\pi_{k}^{i}=\pi_{j k}^{i}+\pi_{i k}^{i}$.

The payoffs are discounted by a common discount factor $e^{-r t}$, so that the netpresent value of profits for the leader (firm i) entering at $t_{i}$ with technology $k$ is:

$$
L_{i}\left(t_{i}, k\right)=\int_{t_{i}}^{\infty} e^{-r t} \pi_{i k}^{i} d t-K^{L}\left(t_{i}, k\right)=\frac{e^{-r t_{i}}}{r} \pi_{i k}^{i}-K^{L}\left(t_{i}, k\right) .
$$

Here, we use the exponentially declining development cost function, $K\left(t_{i}, k\right)=$ $K_{0} e^{\lambda_{k} t_{i}}+K_{i k}$, with $\lambda_{k}>r$.

Similarly, the payoff to firm $i$ if firm $j$ enters with technology $k$ at $t_{j}$ is:

$$
F_{i}\left(t_{j}, k\right)=\frac{e^{-r t_{j}}}{r} \pi_{i k}^{j}-K^{F}\left(t_{i}, k\right)
$$

As firm $i$ maximizes its payoff, the net-present value of profits for the leader (firm i) entering at $t_{i}$ with the best technology available is:

$$
L_{i}\left(t_{i}\right)=\max _{k=1,2}\left[\frac{e^{-r t_{i}}}{r} \pi_{i k}^{i}-K^{L}\left(t_{i}, k\right)\right] .
$$

For simplicity, let us assume that the payoff to firm $i$ if firm $j$ wins with technology $k$ is independent of $k$; that is,

$$
F_{i}\left(t_{j}\right)=F_{i}\left(t_{j}, 1\right)=F_{i}\left(t_{j}, 2\right)
$$

This means that for both firms $\pi_{i 1}^{j}=\pi_{i 2}^{j}$ and $K^{F}\left(t_{i}, 1\right)=K^{F}\left(t_{i}, 2\right)$; a follower earns the same level of profit, regardless as to the technology adopted by the market leader. 
The market demand in each period is 1 unit at a constant price of 1 . We assume that firms share the market equally; the profits before and after entry are

$$
\pi_{i}^{0}=\left(1-c_{i}^{0}\right) / 2, \pi_{i k}^{i}=\left(1-c_{i k}^{i}\right) / 2, \pi_{i k}^{j}=\left(1-c_{i k}^{j}\right) / 2
$$

where $c_{i}^{0}, c_{i k}^{i}$ and $c_{i k}^{j}$ are the costs corresponding to the old and new technology cases.

Several points are worth noting here in relation to this example and the analysis of the model in the paper. Firstly, the follower's payoff function $F_{i}(t)$ is a decreasing function of the leader's entry and the parameters are such that the payoffs are always positive. This means that Assumptions 5 and 6 are satisfied, and that we can apply the framework developed. Secondly, the three curves $L_{1}(t), L_{2}(t)$, and $F_{1}(t)=F_{2}(t)$ - in Figure 6 are all derived using equations (8) - (9). In this way, we are able to construct an entry game with non-monotonic leader payoff functions with only a slight augmentation to an established example in the literature. Moreover, we are able to use this example to illustrate our novel blocking equilibrium (with two iterations).

\section{Appendix B}

\section{Proof of Proposition 2}

This proof consists of four parts: A, B, C, and D. In Part A we show that all SPE with positive entry times must belong to either $A_{1}(0, T)$ if firm 1 enters first or $A_{2}(0, T)$ if firm 2 enters first. In Part $\mathrm{B}$ we prove that for $i=1,2$ there exists a unique $t_{i}^{*}$, given by (3), at which either $L_{i}(t)$ is maximized over $A_{i}(0, T)$ or $t_{i}^{*}=0$ when $A_{i}(0, T)=\emptyset$. Part $\mathrm{C}$ shows that firm 1 entering at $t_{1}^{*}$ is a unique SPE as it delivers the highest possible equilibrium payoffs to both the leader and the follower when $A_{1}\left(t_{1}^{*}, T\right)=\emptyset$ and $L_{1}\left(t_{1}^{*}\right)>F_{1}\left(t_{1}^{*}\right)$. Part D proves that if $A_{1}\left(t_{1}^{*}, T\right)=\emptyset$ and $L_{1}\left(t_{1}^{*}\right) \leq F_{1}\left(t_{1}^{*}\right)$ then there are two SPE with firm 1 entering at $t=t_{1}^{*}$ and firm 2 entering at $t=t_{2}^{*}$.

(A) As a preliminary step, let us prove all SPE with positive entry times must belong to either $A_{1}(0, T)$ if firm 1 enters first or $A_{2}(0, T)$ if firm 2 enters 
first. Assume, on the contrary, that there is an SPE with a positive entry time $t_{i}^{*} \notin A_{i}(0, T)$. It must be the case that both NPL and NPF conditions are satisfied. If condition NPL is not satisfied, the leader (player i) will have an incentive to enter earlier at $\tau$. On the other hand, if condition NPF is not satisfied, the follower (player j) will have an incentive to preempt the leader (player i) and enter slightly earlier, as in Fudenberg and Tirole (1985). Neither of these situations are possible in equilibrium. Consequently, there is a contradiction, proving the statement that all SPE with positive entry times must belong to either $A_{1}(0, T)$ if firm 1 enters first or $A_{2}(0, T)$ if firm 2 enters first.

(B) Next, let us prove that $t_{i}^{*}$ for $i=1,2$ is given by (3). Specifically, there exists a unique $t_{i}^{*}$ at which either $L_{i}(t)$ is maximized over $A_{i}(0, T)$ or $t_{i}^{*}=0$ when $A_{i}(0, T)=\emptyset$. When $A_{i}(0, T)=\emptyset$, entering at $t_{i}^{*}>0$ can not be an SPE; so the only potential entry time for player $\mathrm{i}$ is $t_{i}^{*}=0$. As neither abstaining from entering nor entering later is beneficial, the SPE is either $t_{1}^{*}=0$ or $t_{2}^{*}=0$ or both.

Now consider the situation when $A_{i}(0, T)$ is not empty. Let us prove the existence of the solution to this problem of maximizing $L_{i}(t)$ over $A_{i}(0, T)$ when $A_{i}(0, T) \neq \emptyset$. Note that set $A_{i}(0, T)$ is bounded because $\widehat{T}_{i}$ is finite, where $\widehat{T}_{i}$ is the time at which $L_{i}(t)$ reaches its global maximum (Assumption 4 ). We need to show that set $A_{i}(0, T)$ always contains its supremum. Assume that it does not. This means that there is a sequence $\left\{t_{k}\right\}$ contained in $A_{i}(0, T)$ that converges to some limit $t_{i}^{*}$ that is not contained in set $A_{i}(0, T)$. This requires that either NPL or NPF is not satisfied for $t_{i}^{*}$. As sequence $\left\{t_{k}\right\}$ belongs to $A_{i}(0, T)$, it means that both NPL and NPF hold for sequence $\left\{t_{k}\right\}$. As both $L_{i}(t)$ and $F_{j}(t)$ are continuous functions, it means $t_{i}^{*}$ also belongs to $A_{i}(0, T)$. This leads to a contradiction, proving existence.

The uniqueness follows immediately from the way set $A_{i}(0, T)$ is constructed. If two entry times were to maximize $L_{i}(t)$ over $A_{i}(0, T)$, then the later time would not belong to $A_{i}(0, T)$.

Next, let us show that if $t_{i}^{*}=\arg \max _{t \in A_{i}(0, T)} L_{i}(t)$, it is also the case that $t_{i}^{*}=$ $\arg \max _{t} A_{i}(0, T)$ when $A_{i}(0, T) \neq \emptyset$. Assume the opposite that $t_{i}^{*} \neq \arg \max _{t} A_{i}(0, T)$. If $t_{i}^{*}<\arg \max _{t} A_{i}(0, T)$, then $t_{i}^{*}$ does not maximize the leader's payoff over $A_{i}(0, T)$. If $t_{i}^{*}>\arg \max _{t} A_{i}(0, T), t_{i}^{*}$ does not belong to $A_{i}(0, T)$. Both situa- 
tions lead to a contradiction. We have now shown that $t_{i}^{*}=\arg \max _{t} A_{i}(0, T)$, concluding the proof of Part B.

(C) Next, we prove that firm 1 entering at $t_{1}^{*}$ given by (3) is a unique SPE when $A_{1}\left(t_{1}^{*}, T\right)=\emptyset$ and $L_{1}\left(t_{1}^{*}\right)>F_{1}\left(t_{1}^{*}\right)$. Note that in this case $t_{1}^{*}>t_{2}^{*}$. First, in part $\mathrm{A}$ we proved that there is no equilibrium with entry time $\tau>t_{1}^{*}$.

Second, let us prove that firm 1 entering at $t_{1}^{*}$ is an SPE. In part B we proved that $t_{1}^{*}$ maximizes $L_{1}(t)$ over $A_{1}(0, T)$. This means the leader gets the highest possible equilibrium payoff and has no incentive to deviate. The follower has no incentive to deviate as well as $t_{1}^{*}$ belongs to $A_{1}(0, T)$ and $A_{1}\left(t_{1}^{*}, T\right)=\emptyset$.

Third, let us prove that there is no equilibrium with entry time of firm $1 \tau<t_{1}^{*}$. As $t_{1}^{*} \in A_{1}(0, T)$, entering at $t<t_{1}^{*}$ is strictly dominated by entering at $t_{1}^{*}$.

Finally, let us prove that there is no SPE with firm 2 entering at any $t$. Part A guarantees that entering at $t>t_{2}^{*}$ can not be an SPE. The follower also has no incentive to enter at $t \leq t_{2}^{*}<t_{1}^{*}$ as $t_{1}^{*}$ belongs to $A_{1}(0, T)$, which means NPF condition is satisfied and for firm 2 waiting dominates entering.

(D) Let us prove that if $A_{1}\left(t_{1}^{*}, T\right)=\emptyset$ and $L_{1}\left(t_{1}^{*}\right) \leq F_{1}\left(t_{1}^{*}\right)$ then there are two $\mathrm{SPE}$ with firm 1 entering at $t=t_{1}^{*}$ and firm 2 entering at $t=t_{2}^{*}$. Consider an SPE where firm $\mathrm{i}$ is the leader and firm $\mathrm{j}$ is the follower $(i, j=1,2$ and $i \neq j)$.

First, given $t_{i}^{*} \in A_{i}(0, T)$, if the follower deviates by entering at some time $\tau<t_{i}^{*}$, it will get a payoff of $L_{j}(\tau)<F_{j}\left(t_{i}^{*}\right)$. If it deviates by entering at $t_{i}^{*}$, it will get a payoff of $\left(L_{j}\left(t_{i}^{*}\right)+F_{j}\left(t_{i}^{*}\right)\right) / 2$, which is not greater than $F_{j}\left(t_{i}^{*}\right)$. If the follower enters at $t>t_{i}^{*}$, there will be no change to the equilibrium outcome. Consequently, there is no profitable deviation for the follower.

Second, in part A we proved that there is no equilibrium with the leader entering at $\tau>t_{i}^{*}$. Given $t_{i}^{*} \in A_{i}(0, T)$, if the leader deviates by entering earlier at some time $\tau<t_{i}^{*}$, it will get a payoff of $L_{i}(\tau)<L_{i}\left(t^{*}\right)$. There is no profitable deviation for the leader.

There is no other equilibria as entering at $t_{i}^{*}$ dominates entering at any other time. Consequently, we have proved that there are two equilibria. This observation concludes the proof of this Proposition. 


\section{Proof of Proposition 3}

Let us first consider case $(2)$ when $A_{1}\left(t_{1}^{*}, T\right) \neq \emptyset, A_{2}\left(t_{2}^{*}, t_{1}^{*}\right)=\emptyset$ and $t_{2}^{*}=\bar{T}_{2}$. In this scenario relative to $(\mathrm{D})$ in Proposition $2, A_{1}\left(t_{1}^{*}, T\right) \neq \emptyset$. This means firm 1 entering at $t=t_{1}^{*}$ is not an equilibrium as at $t_{1}^{*}$ firm 1 has an incentive to wait. As a result firm 2 will have incentive to block firm 1's entry. Note also that for the same reason as in part (D) of Proposition 2, entering by either firm i at any other time $t \neq t_{i}^{*}$ is not an SPE. Consequently, there exists only one SPE with firm 2 entering at $t=t_{2}^{*}$.

Consider instead case (3) when $A_{1}\left(t_{1}^{*}, T\right) \neq \emptyset$ and $A_{2}\left(t_{2}^{*}, t_{1}^{*}\right) \neq \emptyset$. In this case in comparison with case $(2)$, neither entering by firm 1 at $t_{1}^{*}$ nor entering by firm 2 at $t_{2}^{*}$ is an equilibrium. Firm 1 has strong incentives to enter even earlier (and block firm 2's entry) as firm 2 has incentives to wait at $t_{2}^{*}$. Consequently, there is a unique SPE with firm 1 entering at $t_{1}^{* *}$.

Finally consider case (1) when $A_{1}\left(t_{1}^{*}, T\right) \neq \emptyset, A_{2}\left(t_{2}^{*}, t_{1}^{*}\right)=\emptyset$ and $t_{2}^{*}<\bar{T}_{2}$. In this case $t_{2}^{*}=\widetilde{T}_{1}$, which in turn imply that $L_{1}\left(\widehat{T}_{2}\right)>F_{1}\left(\widehat{T}_{2}\right)$ and $L_{2}\left(\widehat{T}_{2}\right)<F_{2}\left(\widehat{T}_{2}\right)$. There is no pure strategy equilibrium as at $\widehat{T}_{2}$ firm 1 's best response to the entry by firm 2 is to enter slightly earlier. Firm 2 would prefer to follow if firm 1 enters, but if it does so, firm 1 would also have an incentive to wait, as its leader's payoff is increasing with time. This observation concludes the proof of this Proposition.

\section{References}

M. Alipranti, C. Milliou, and E. Petrakis. Timing of technology adoption and product market competition. International Journal of Industrial Organization, 29:513-523, 2011.

M. Alipranti, C. Milliou, and E. Petrakis. On vertical relations and the timing of technology adoption. Journal of Economic Behavior and Organization, 120: 117-129, 2015.

Axel Anderson, Lones Smith, and Andreas Park. Rushes in large timing games. Econometrica, 85(3):871-913, 2017. 
Rossella Argenziano and Philipp Schmidt-Dengler. Inefficient entry order in preemption games. Journal of Mathematical Economics, 48(6):445-460, 2012.

Rossella Argenziano and Philipp Schmidt-Dengler. Competition, timing of entry and welfare in a preemption game. Economics Letters, 120(3):509-512, 2013.

Rossella Argenziano and Philipp Schmidt-Dengler. Clustering in $n$-player preemption games. Journal of the European Economic Association, 12(2):368-396, 2014 .

Francis Bloch, Simona Fabrizi, and Steffen Lippert. Learning and collusion in new markets with uncertain entry costs. Economic Theory, 58(2):273-303, 2015.

Catherine Bobtcheff and Thomas Mariotti. Potential competition in preemption games. Games and Economic Behavior, 75:53-66, 2012.

Prajit K. Dutta, Saul Lach, and Aldo Rustichini. Better late than early: Vertical differentiation in the adoption of a new technology. The Journal of Economics and Management Strategy, 4:449-460, 1995.

Glenn Ellison and Sara Fisher Ellison. Strategic entry deterrence and the behavior of pharmaceutical incumbents prior to patent expiration. American Economic Journal: Microeconomics, 3(1):1-36, 2011.

Drew Fudenberg and Jean Tirole. The fat-cat effect, the puppy-dog ploy, and the lean and hungry look. American Economic Review, 74(2):361-366, 1984.

Drew Fudenberg and Jean Tirole. Preemption and rent equalization in the adoption of new technology. The Review of Economic Studies, 52(3):383-401, 1985.

Drew Fudenberg and Jean Tirole. Game Theory. Cambridge: MIT Press, 1991.

Alberto Galasso and Mihkel Tombak. Switching to green: The timing of socially responsible innovation. Journal of Economics \& Management Strategy, 23(3): 669-691, 2014.

Ricard Gil, Jean-Francois Houde, and Yuya Takahashi. Preemptive entry and technology diffusion: The market for drive-in theaters. 2019. 
Kenneth Hendricks. Reputations in the adoption of a new technology. International Journal of Industrial Organization, 10(4):663-677, 1992.

H.A. Hopenhayn and F. Squintani. Preemption games with private information. Review of Economic Studies, 78(2):667-692, 2011.

Heidrun Hoppe. Second-mover advantages in the strategic adoption of new technology under uncertainty. International Journal of Industrial Organization, 18 (2):315-338, 2000.

Heidrun Hoppe. The timing of new technology adoption: Theoretical and empirical evidence. The Manchester School, 70(1):56-76, 2002.

Heidrun Hoppe and Ulrich Lehmann-Grube. Second-mover advantages in dynamic quality competition. Journal of Economics \& Management Strategy, 10(3):419433, 2001.

Heidrun Hoppe and Ulrich Lehmann-Grube. Innovation timing games: A general framework with applications. Journal of Economic Theory, 121:30-50, 2005.

Michael Katz and Carl Shapiro. R \& D rivalry with licensing or imitation. The American Economic Review, 77(3):402-420, 1987.

Robert E. Krider and Charles B Weinberg. Competitive dynamics and the introduction of new product: The motion picture timing game. Journal of Marketing Research, 35(1):1-15, 1998.

Jordi McKenzie and Vladimir Smirnov. Blockbusters and market expansion: Evidence from the motion picture industry. Journal of Cultural Economics, 42: 341-352, 2018.

Andreas Park and Lones Smith. Caller number five and related timing games. Theoretical Economics, 3:231-256, 2005.

Jennifer Reinganum. On the diffusion of new technology: A game-theoretic approach. Review of Economic Studies, 153:395-406, 1981a. 
Jennifer Reinganum. Market structure and the diffusion of new technology. Bell Journal of Economics, 153:618-624, 1981b.

Michael Riordan. Regulation and preemptive technology adoption. RAND Journal of Economics, 23(3):334-349, 1992.

Philipp Schmidt-Dengler. The timing of new technology adoption: The case of MRI. 2006.

L. Simon and M. Stinchcombe. Extensive form games in continuous time. Econometrica, 57(5):1171-1214, 1989.

Vladimir Smirnov and Andrew Wait. Staged financing with a variable return. B.E. Journal of Theoretical Economics, 7(1):1-26, 2007.

Vladimir Smirnov and Andrew Wait. Innovation in a generalized timing game. International Journal of Industrial Organization, 42:23-33, 2015.

G.J. Tellis and P.N. Golder. First to market, first to fail? Real causes of enduring market leadership. Sloan Management Review, 37:65-75, 1996.

Ngo Van Long. A Survey of Dynamic Games in Economics. Singapore: World Scientific, 2010. 